\title{
Transitioning from Transmedia to Transreality Storyboarding to Improve the Co-Creation of the Experience Space
}

\author{
Jazz Rasool \\ Ravensbourne University London \\ UK \\ j.rasool@rave.ac.uk
}

\author{
Judith Molka-Danielsen \\ Molde University College \\ NO \\ j.molka-danielsen@himolde.no
}

\author{
Carl H. Smith \\ Ravensbourne University London \\ UK \\ c.smith@rave.ac.uk
}

\begin{abstract}
Transmedia storytelling is a digital based marketing approach in present day consumer markets. Typically applied to spanning or segueing stories or experiences across media such as film, books, comics and video-games to reach broader target audiences, often triggering a narrative, into which customers can participate and co-create the narrative. Common aims at customer engagement have been through shared stories on present day social media. However, for the creative-consumer, sharing on social media falls short of fully immersive storytelling ecology. Creatives (traditional designers and consumers) would benefit through tools and processes for incrementally expanding dimensions, mediums, fidelity, and shared interactions and senses across multiple media and interactive realities. This paper presents use cases of Transreality Storyboarding Framework (TSF), a design framework that affords creation of experience spaces for consumer-product engagement. Further, we propose a TSF app, to allow non-expert designers/everydayconsumers to contribute to storytelling, participation and production of product experience spaces.
\end{abstract}

\section{Introduction}

Increased use of Augmented and Virtual Reality experiences in branding and marketing have led to engaging customer interactions with products and services within the immersive reality chosen but have failed to capitalize on higher fidelity encounters and immersion that come from linking experiences across devices and realities. Consumer facing tools or apps that allow creation of Augmented or Virtual reality experiences are still in nascent development and dissemination stages. It is easy to take a photograph or shoot a video and share over a network but not so easy to create an object or experience and deliver it as a shareable AR output or VR interaction. Even if this is possible there is no user experience friendly framework to help people produce content, let alone span a storytelling process across devices.
Traditional methods of storytelling that frame stages of a media experience usually rely on storyboarding, a process that attempts to encapsulate a narrative path in 2-dimensional frames with representative illustrations that relate to a story plot or feature point. For emerging immersive realities though, in addition to visual, script and audio references, there is touch based content as well as smell and taste related interactions possibly mediated by sensors for biometric signals such as heart rate or skin resistance. Such a spectrum of sensory or sensor channels need to be included in the story design in addition to the media and realities being worked with. A third factor is that for every sensory channel, media, reality or device, a story is communicated through there will have to be an interface, menu, icons or affordances through which interactions will take place. The interface must be able to be adaptive to content that covers different senses and sensor data as well as streaming content. Transmedia needs an upgrade in its storyboarding tools so it can work across realities and sensory channels and streams. It needs a framework to storyboard across realities. A Transreality Storyboarding Framework (TSF) has been created to facilitate this and to create workflows and strategies to raise the fidelity and engagement possible in marketing and branding campaigns [1].

A key challenge facing marketing initiatives that want to migrate to these new calls for enriched fidelity, presence and atmosphere will be the need to address how branding and marketing departments transition between Transmedia to Transreality based design and production strategies or workflows.

This paper is laid out as follows. In Section 2 we provide a theoretical review of trans-mediation, transmedia and transreality based storytelling. We describe the emerging need to transition to a transreality based marketing approach. In Section 3, we describe how marketing approaches can transition to transreality storyboarding through application of the TSF. In Sections 4 we describe our methodology. In 5 we present a use case in which TSF was applied in marketing. In Section 6 we reflect on the implications 
for marketing and future opportunities for marketing new products and services. In Section 7 we propose the basic components of a TSF App that would afford everyday-consumers the ability to contribute to the cocreation of the storytelling process. Finally, in Section 8 we give concluding remarks and offer suggestions for future directions for transreality storyboarding and its engagement of co-creative consumers.

The authors propose there is a novelty in the research question that we aim to address in this paper, specifically: how can immersive reality experiences be efficiently and effectively produced by individual creatives or creative project teams to deliver coherent and engaging storytelling arcs that span different media, immersive realities and related technologies?

\section{A Review of Trans-mediation, Transmedia and Transreality Based Storytelling}

Theories and former research in trans-mediation that are founded in media and communications psychology form a basis for our research in transreality storyboarding. Research defines presence as a sense of being in an environment and, further, telepresence is that one feels presence in a technology mediated environment [2][3]. Important factors in telepresence are vividness and interactivity [2]. Further, factors of vividness are sensory breadth and sensory depth. Sensory breadth is the number of sensory dimensions, and sensory depth is the resolution of each channel. A sense of being at the beach may be generated by of combination of many sensory inputs (e.g. the smell of the sea, the feeling of the sand, hearing the cry of a seagull, the taste of ice cream, etc.) How these sensory inputs come together, that is how they are mediated, creates the sense of vividness.

Interactivity has to do with how the user can map and make persistent their actions into the mediated environment [2]. Biocca points out "The Cyborg's Dilemma", describing the paradox that as virtual technologies interfaces advance that the user adapts and changes to them in an unnatural way. She outlines three premises that are (1) humans have been augmenting their bodies with technology for quite some time (e.g. clothing, glasses, etc.); (2) advances in technologies tighten the relationship of our minds and bodies to technology; and (3) the effect of tight coupling is that the mind becomes adapted to the mediated body. In brief the experience has effect that the user becomes used to their new identity in the mediated environment $[4, \mathrm{p}$. 37].

Some describe presence as a subjective measure of a user's feeling of being there, while a user's immersion can be measured objectively as a level of fidelity against another experience of a mediated environment [5]. More interesting is that little is known about the effects of presence on a user once it is invoked. Lombard and Ditton present six conceptual definitions of presence as variables: social richness (intimacy and immediacy), realism (accuracy), transportation (or place), immersion (perceptual and psychological), social actor within medium (e.g. your avatar) and medium as social actor (e.g. robot or AI-chat bot). They point out that objective measures could be made of these variables to assess users' psychological and behavioral response during media use [6].

Designers and marketers would alike benefit from understanding the effects of presence on factors like enjoyment and performance. Recent research by Bowman looks at the affective demands on users at playing video games [7][8]. He points out that the meaningful games have great stories, allow users to relate to users in the game and result in emotional experiences, and result in feelings of appreciation. He found that meaningful games were memorable. In juxtaposition, the user's rating of a game as meaningful had nothing to do with their rating of enjoyment. Enjoyment was related to competence and efficacy of gameplay. He stated that designers will need to choose to focus on the narrative experience (related to appreciation), the challenge experience (related to enjoyment) or both [7] [8].

Finally, few studies have explored how psychological processes are triggered by transmediated technologies (some that have include[9][10]). Busselle and Bilandzic developed a scale for measuring narrative engagement involving four factors: narrative understanding (comprehension), attentional focus (as opposed to the mind not wandering), emotional engagement (feeling for and with characters), and narrative presence (constructing new worlds) [9]. As part of the process of narrative engagement, people will create mental models that are cognitive structures of how one relates to and represents some part of the represented world [11]. They found the support of narrative presence was particularly important for achieving narrative engagement [11].

Pressgrove and Bowman found that narrative engagement is critical to impacting prosocial attitudes towards the experienced mediated content. In their study showing 360 videos designed to encourage actions to prevent malaria, they found that people that wore head mounted displays felt a significantly higher sense of presence. However, the effects of feeling present, did not lead them to feel more engaged in the story. They also found that the type of immersive technology only helped users to feel closer to the physical location of the narrative, but did not help them align with the 
behavioral intensions of the narrative [10]. Their study and other research support [12] that immersive technologies may help users to feel "in a mediated place" (presence) but not necessarily "in a mediated story" (narrative engagement) [10, p. 9]. We suggest that in many trans-mediated solutions will have historically applied a Transmedia Storytelling design approach. However, our proposed TSF framework will aid designers and marketers to support narrative engagement.

Transmedia Storytelling was first introduced as a multi-channel or multi-platform approach to enhance storytelling. It is described as a co-creation model for the adaption of content to be consumed across media [13] [14]. Others define Transmedia Storytelling as a multi-channel paradigm, and make the distinction of structures in that, a story as adapted in different channels is a cross-media expression, and the story if spread through different channels is a transmedia phenomena [15]. Both of these definitions of transmedia storytelling focus on the output expression of the story. Additionally, in the creation of stories the story may be disjoint over several media including graphic novels, film, video games, etc. where the design processes have no connection with each other [14].

The disconnection of a story told across independent media can be illustrated using Robert Pratten's illustration showing affordances achieved through Transmedia approaches to storytelling shown in Figure 1(a) and (b) [16]. In Figure 1(a) the typical media franchise approach to promoting a story through different media is without integration or coherence whereas in Figure 1(b), the Transmedia approach creates media designed to work with each other for a more consistent and sustainable story across channels.

The Transmedia approach enhances the story by engineering synergy across the media it is promoted across. Although transmedia approaches allow greater synergy of storytelling elements communicated across different media, they need to be updated to take into account how experiences are expressed across different sensory dimensions of reality. Specifically, such elements moving across everyday reality through publication or device based 2D, 3D and higher incarnations including those with sensory affordances beyond audiovisual content such as touch or haptic based interactions. So, an alternate version of Figure 1(b) could have some additional extensions to cover a story being told across different realities, interactions and sensory channels.

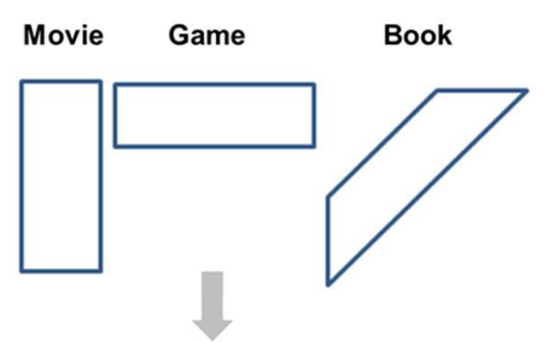

Typical Media Franchise

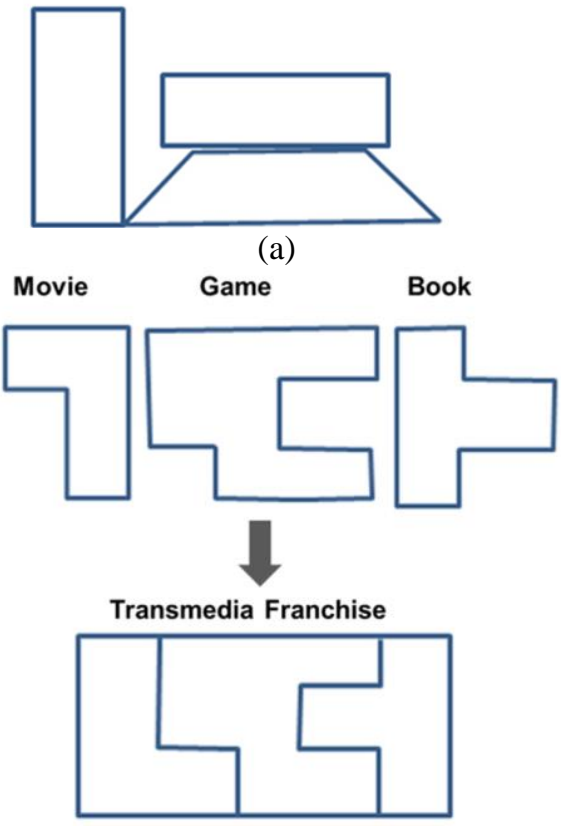

(b)

Interactive Multisensory Augmented Movie VR Game Reality Book

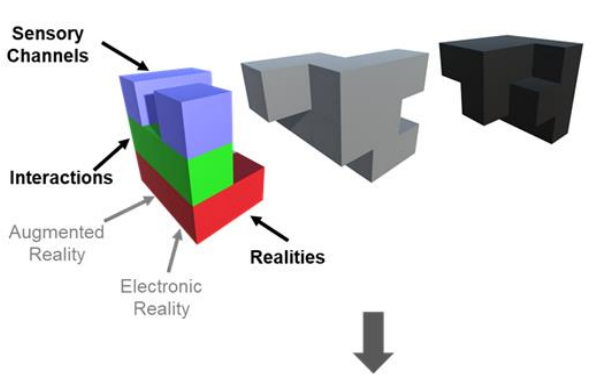

Transreality Experience

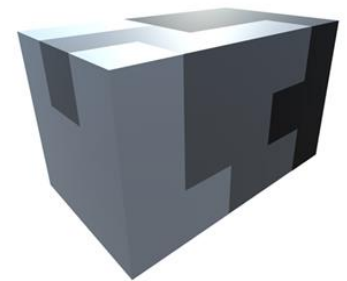

(c)

Figure 1. Comparing Typical, Transmedia and Transreality approaches to telling stories. 
In Figure 1(c) the authors illustrate Transmedia extensions to show how an interactive movie can be represented in Electronic Reality as a streamed film but also as an Augmented Reality experience that can have image targets on it that could bring it to life within a viewer's physical space.

The movie could be made interactive through the image targets and engage specific sensory channels through visual animation, audio narratives and even access sensor data from the environment or internet to augment the movies content and flow. Each of these realities, interfaces and sensory channels would be in turn aligned with a multisensory, higher fidelity game adaptation of the movie that could be: a real world inperson activity, a Virtual Reality video game, or an immersive high presence and enriched atmospheric book experience that has Augmented or Virtual Reality content. The content generated depends on the device the experience is being created through.

Advancing the Transmedia process of Figure 1(b) results in the Transreality storytelling structure of Figure 1(c). This transition is advanced by mapping three roles of additional immersive dimensions: (1) Realities (2) Interactions and (3) Sensory Channels and the way these roles determine how a movie, game and book fit into one another's contributions to an overall higher fidelity, more deeply engaging, extended, sustainable and coherent narrative.

Audiences for Transreality stories would typically be defined by the realities they would experience the story in, what interactive affordances were available and the sensory fidelity provided by devices or environments to express the story.

Just as how Transmedia approaches can provide more whole and engaging experiences by how stories are told in an aligned way across media, Transreality approaches ensure the experience is whole, engaging and has appropriate fidelity when experienced through different realities, interactions and sensory channels. So, if Transmedia approaches can be considered an augmentation, enhancement or evolution of typical media storytelling franchises then Transreality experiences can be considered an evolution of Transmedia. Transreality storyboarding approaches update Transmedia workflows to cater for technologies and platforms that create immersive experiences which span across and through many realities associated with immersive productions, especially Augmented, Virtual and Mixed Reality experiences. The structure and scaffolded flow can facilitate consumers to be guided in creating their own content for existing 'new media' storytelling and its emerging future generation of immersive realities.

Historically, transmedia storytelling has had a strong focus on authorship [17]. However, there is a lack of tools to allow designers, including co-creative consumers, ways of evaluating and contributing to experiences in transmedia designs. We propose therefore, that there is a gap in the research and supportive tools to afford co-creative consumers with the ability to envision and contribute to a story through a co-created user perceived experience space.

Museums have been leaders in applications of transmedia storytelling. They, in the curation and design of artifacts, increasingly apply multi-channel approaches [18][19][20][21].

\section{Transitioning from Transmedia to Transreality Storyboarding}

Marketing initiatives that respond to new calls for enriched fidelity, presence and atmosphere must understand what novel mindsets, techniques and technologies can foster design, production and promotion of their campaigns. How campaigns transition between utilising Transmedia driven projects to workflows driven by Transreality methodologies must be clarified. From Figure 1(c), transitions in operations, design and production must centre on adapting stories across different realities, interactions or interfaces as well as through tailored sensory experiences or content augmented by data from channels linked to sensors or datastreams derived from networks such as the Internet. Advertisers, Branding agents and Marketing Campaign managers have to codify stories using these mindsets through techniques that adopt tools to design, track and deploy transreality initiatives. The authors have already created techniques and designed tools to gather requirements for producing Transreality experiences. These use Participatory Design approaches, working with stakeholders to establish what realities a story will be told within and across, what sensory experiences or data will be gathered and delivered as well as clarify what interactive affordances will be provided through aligned interfaces in software, hardware or through real world tools or props. The aim with Transreality Storyboarding is to generate a response from a consumer through a targeted experience, a response that triggers sharing of the experience with others or initiates an aligned shift in the consumer's behavior or activity, one that ethically enhances brand awareness or engages commercial or community engagement with an enterprise.

The Transreality Storyboarding Framework (TSF) was introduced in a previous paper [1]. TSF has three components that contribute to the creation of crossreality holistic narrative alignment. The components are:

1. Eight Realities Design Methodology (ERDM) maps eight fundamental realities often engaged with in 
the creative process to mediate a creative product. These are: (1) Imaginary reality that is an internalized view of the real world, (2) Everyday reality of the real world without aids, (3) Captured/drawn reality such as paper illustration, photographs, video capture or 3D scans (4) Computer Aided Design/CGI reality, (5) 3D Printed reality, (6) Augmented Reality (7) Virtual Reality and (8) Holographic/Extended/Mixed Reality.

2. PAIRS component identifies needed user interface support for connection/interactions with the learning environment (passive, active, interactive, responsive and shared-PAIRS). Considerations need to be identified for a design of the interface between mediums (realities) and media (sense) which afford specific interactions that users will have with the desired generated experiences.

3. Contextology is a component that profiles the user-senses (hearing, seeing, touching, etc.) that are intended to be activated during an interaction [22]. It is critical that sensory channels that need to be targeted are aligned with media assets that are created for them.

An example of a first stage of mapping a prospective Transreality experience is discussed in the following section that discusses a project that used the framework for showcasing an actual museum exhibition.

\section{Methodology}

This section describes our methodology for development of the element components of the TSF, and the trialing and assessment of the initial framework. Our approach can be loosely described as an IDEO Design Thinking (ideo.com) approach. The authors experience gained from early projects formed (1) the driving questions. Interactions with users, for example at museums, (2) contributed to our greater understanding of the research problem. Next, we (3) built the prototype of the TSF. Through trialing the framework, we (4) gathered feedback from users experimenting with it. Finally we have (5) reflected and transferred our understanding back into the proposed design of a TSF app that may better afford non-expert designers to contribute to the design and production of product experience spaces.

\subsection{Pilot of the TSF Components}

In 2019, to celebrate the 500th anniversary of Leonardo da Vinci's death an exhibition was held at the Peltz Gallery and the Victoria and Albert Museum in London, UK and the Museo Galileo in Florence, Italy [23].

The elements that would eventually comprise the TSF were first informally piloted in 2018-2019 by
Birkbeck College and Ravensbourne University. This was through producing an installation to promote the 500th anniversary of Leonardo Da Vinci's death by showing his designs for perpetual motion machines as a story arc that spanned illustration, 3D Computer Modelling and Animation, Video, 3D Print, Augmented Reality and Mixed Reality. Those working with the process were experts in media curation.

A matrix tabulating the curation process of the exhibitions is shown in Figure 2. The matrix outlines an experience that will be communicated to museum and gallery visitors, one where they will sensorially see experiences across all realities, including captured voice interaction and audio narratives that people hear. Enhanced interactions will happen through touch and hand gestures. Although it was planned to use streamed data about a person's physical wellbeing using signals from body temperature and heart rate sensors, the final installation did not deploy this, however another project that proposed using the same fidelity of experience did use all affordances, using a mixed reality approach to enhance learning and development [24, 25].

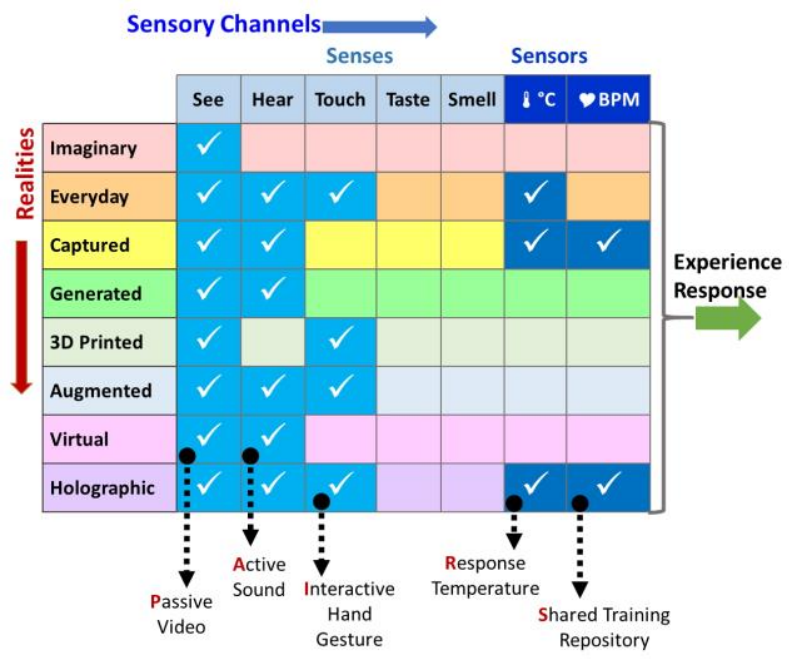

Figure 2. TSF component interactions [1]

\subsection{Trial and Feedback of TSF with Students}

Subsequently the elements were formally composed into a framework, and the TSF was trialed in 2019 in the EU funded project, Augmented Reality for European Universities (AR4EU) and an online class it held as part of its AR curriculum. In the AR4EU session, twelve class participants were divided into four groups and were guided through the imagining of a story and how it would be told through use of multi-track storyboarding, to create a coherent and engaging story arc spanning experiences delivered as 2D Illustration, Video, 3D Print, Augmented Reality and Virtual 
Reality. The students gave feedback that the TSF contributed to brainstorming, visualization and organization of the design elements. But, commented that it was difficult to work with the components as supported through spreadsheets.

In a second trial, TSF was introduced to students of the Hobbs 3D Academy to enable them to effectively take aspirations of prospective clients in Architecture, Engineering and Construction projects and simulate their realization. This was done through a story arc that would portray the aspiring design using scriptwriting and immersive reality storyboarding. The story plan was then represented through interlinked assets of 3D Models, Video, 3D Prints, Augmented Reality and Virtual Reality with a fully interactive guided story delivered through a Unity game engine app. The Hobbs students found it a stretch to organize the process of telling their story, especially in its storyboarding, organizing documentation and versioning of assets as well as overall project management. In feedback, they felt they could have done with more training in the framework documentation process so more effective accountability of project team members' outputs could have been monitored and sustained. The end results of each project team were interactive game engine experiences that told a story that was more engaging and coherent than if simply composed through free-flowing creative, spontaneous or transactionally carried out tasks. The feedback from both trials indicated a need for a TSF app for non-expert designers that is described further in Section 7.

\section{TSF in Marketing to AEC Clients}

In 2020, The London Mayor's office recognized the need for Architecture, Engineering and Construction industries to improve their workflows and enrich client experiences by ensuring architects and engineers utilised 3D Modelling, Model Making, 3D print and Immersive Reality in a more multi-modal coherent way. It aimed to avoid silo-based workflows that often encountered bottlenecks in performance when moving designs between working roles. This was needed to make projects progress more effectively and efficiently but also ensure clients were shown representations of designs that they were more likely to respond to through immersive promotional experiences. The authors of this paper created an immersive realities curriculum of training for industry professionals that updated their skill sets with immersive reality production workflows to enable them to promote their designs to clients in more engaging, interactive high fidelity experiences using the Transreality Storyboarding Framework [26]. After constructing a matrix to determine the response to be generated by the client experience such as one similar to that in Figure 2, the Transreality Storyboard would be compiled. To facilitate more effective promotion of designs and increase likelihood that marketing campaigns would secure contracts or clients, participants were shown how to storyboard across several tracks of Reality. A track may be seen as a means or pathway of expression. In each track, for example a Virtual Reality track, would be a frame by frame storyboard that would include what scene was being created, what shot in the sequence within it as well as spaces for choosing the types of interactions and the sensory channels or sensor data streams that would be incorporated. Figure 3 (a). Shows an overview of tracks a Transreality Storyboard uses to link experiences across realities of 3D Print, Augmented Reality, Virtual Reality and Augmented Reality with arrows showing flow of experience between components of each reality.

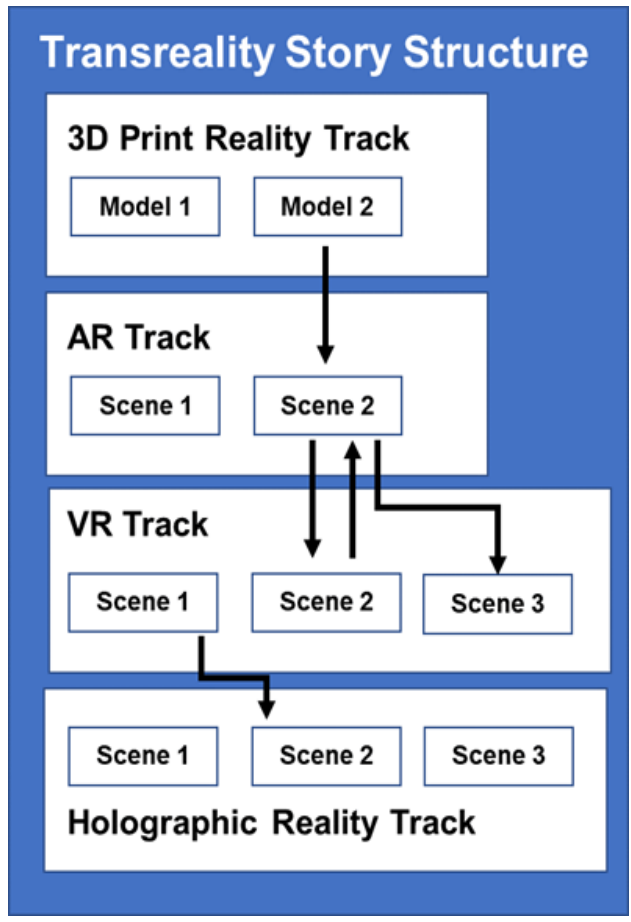

(a)

\section{Augmented Reality Track}

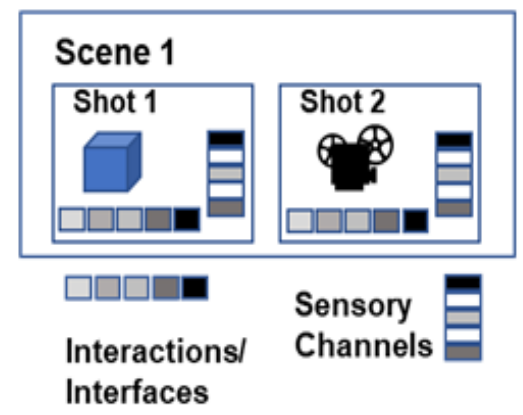


(b)

\section{Figure 3. Transreality Storyboard Structure used in promoting AEC designs to clients}

An example of the detail of the AR Track, showing the first scene's detail, can be seen in Figure 3 (b), indicating the Sensory channels selected and Interactions/Interfaces selected for facilitating the sensory experience in the AR part of the story. Each frame also includes a cell to place content indicative of that component of the experience such as an interactive 3D model or multimedia asset. An example of how a track, its scenes, component shots and constituent sensory and interaction assets might be constructed in a prospective mobile Transreality Storyboarding Framework application is shown in sample wireframes outlined in section 7 later in this paper.

\section{Implications for Marketing Campaigns and Future Opportunities}

Expertise for working with AR and VR technologies are at present out of the reach of most modern day marketing departments. Yet, those providing marketing experiences for institutions or events, would benefit from being able to map their stories across realities, and from the ability to reach different kinds of audiences.

New technologies, changes in people behavior and new choices, have all contributed to a changing economy from agricultural (commodities) and industrial (goods production); to that of service economy and most recently to the emergence of the experience based economy. Experience based economies have the goal to differentiate services into experiences where value is derived from a "product or service" that can be intangible and is "in the moment" experienced content. The "value" of the product or service is realized in the "consumption" of the experience. $[27,28]$

As we transition from a mass marketing approach, beyond transmedia to a transreality approach, we may ask how do we maintain the sense of presence and engagement of the customer. The transition to new marketing approaches is evolutionary. It is not that old forms of marketing are completely replaced. Rather, the transreality approach offers additional affordances to tell a coherently whole story across media realities. The TSF approach bridges the gap to provide experience responses that maintain fidelity and presence that translates into in-the-moment value.

In terms of future opportunities, we have chosen three transmedia experience design companies to collaborate with in order to explore potential ways that the TSF can help them improve their products.

\subsection{Odyssey Works}

Odyssey Works is a transreality experience design company where an immersive real world scenario is constructed for one individual who is positioned at the heart of the performance [29]. The factors that distinguishes Odyssey Works from other transmedia design companies include:

- A performance that is designed for an audience of one.

- The importance of duration: the experience can last days, weeks, or months

- The collective is made up of artists in dozens of disciplines who study the life of one individual and use whatever means necessary to create intimate, meaningful lasting experiences.

- Performances occur not on a stage but are interwoven with the life of their audience of one.

- The intended Output / Experience response: Transformation; most of the participants change jobs, move, make new commitments to loved ones.

- Odyssey Works meta intention is to create truly collaborative teams, effective design, and a positive impact on the world.

\subsection{Waldorf Project}

The Waldorf Project is a genre defying and radical immersive art performance that uses emotional manipulation combined with technologies to develop new ways of implementing algorithmic methods which manipulate a group in order to explore a creative vision [30]. The Project unites the most pioneering practitioners in the worlds of environment design, product design, spatial design, sound design, choreography, textile design, and gastronomy, to engender the birth of a new art form: a rare synergy, a singular collected sensory reaction, or 'sixth sense' of emotion. The Waldorf Project is a radical immersive experience on a grand stage in which art is consumed through all of the senses. The Waldorf Project unites the senses through movement, sound, food, scent, psychology and environment. Key to this concept is the synergy between the various aspects of the event and the imaginations of the creative team. From its genesis eight years ago as a multi-sensory dining experience, the Waldorf Project has evolved into a daring experiment in consciousness transformation. The Waldorf Project also utilizes VR to model the expected flow in the experiences.

\subsection{The Co-Reality Collective}

The Co-Reality Collective is an online party and entertainment service devoted to promoting the next 
phase of reality via parties [31]. They have produced specialized software that enables them to scale up the production of their parties. They are experimenting with the internet as an experimental medium. The average party lasts 8 hours with 16 unique Zoom rooms full of dancing / comedy / intimacy workshops / blind burners / healing dance meditations / a trust disco / live jazz bands / arts \& crafts / aerial, hoop, and fire cabaret performances / real connection.

With the three companies presented in Sections 6.1 - 6.3 we propose to use the TSF to provide the following functionality:

1. Give the audience the opportunity to visualize the experience from various levels of granularity.

2. Allow for the co-creating of the design process and the formation of the experience

3. Improve the Narrative construction of the product.

4. Understand and prototype how a truly mixed reality can be formed through the combination of analogue and digital elements

5. Test experiences before they are manufactured.

\section{Proposal for TSF App for Non-Expert Designers}

A TSF App will help automate the workflow process for the non-expert designer. Ideally the app would be operational through a mobile device.

The workflow process begins by generating lists of the elements that will be input and integrated to create the desired output experience responses. The lists generated are based on the component models: EDRM, Contextology and PAIRS. These are:

1. Reality List - choose the realities that you want to create within and across (e.g. 3D printed and VR).

2. Sensory List - choose the sensors and senses by which the reality will be engaged with, exchanged and enhanced (e.g. seeing and heart rate monitor)

3. Interaction List - For each chosen sense and reality interaction, choose the type of interaction that is desired in the interface (e.g. interactive and shared).

Each of these lists may have multiple and mixed types of elements. The next step will be to apply the lists in a sequence of steps that describe the experience. This step is to transform the lists into scenes and framed 'shots' that are represented in time sequenced grid templates. An example of a scene represented in a grid template is depicted in Figure 2. The TSF grid templates are developed for each scene and its shots in sequence, applying the input lists. An example of how this could be planned is illustrated in Figure 4.

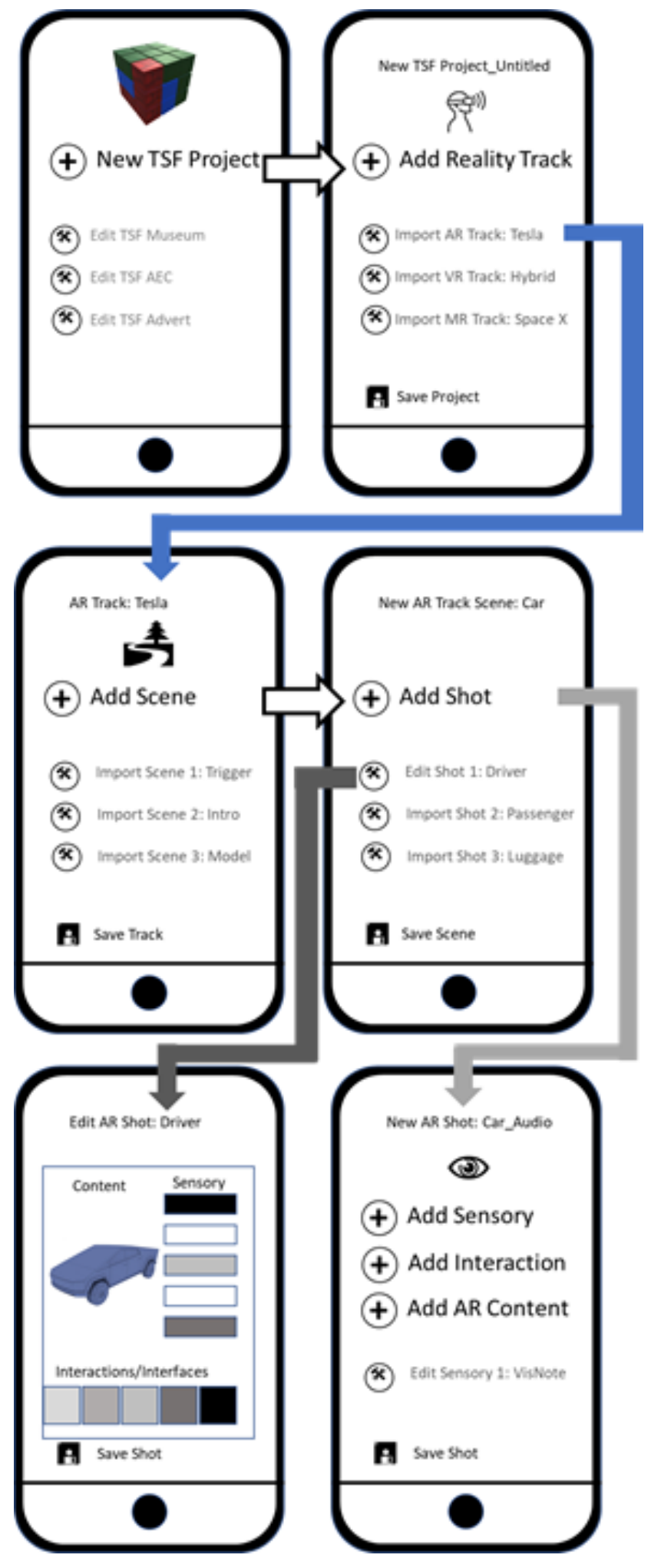

Figure 4. Transreality Storyboard App High Level User Experience Journey 
The TSF App would then take these inputs and produce a Transreality Breakdown Structure (TBS). This term is an adaptation of a commonly used term in project management, that is a Work Breakdown Structures (WBS). A WBS is defined as "a deliverableoriented grouping of project elements which organizes and defines the total scope of the project. Each descending level represents an increasingly detailed definition of a project component." [32] At the lowest level these components may be defined as specific tasks of work to be completed in the life-cycle of the project.

The aim of the TBS is different from the WBS. The lowest component in this structure are not tasks, but rather a scene's shot that is a slice of time in a mixedreality experience response.

Ultimately, the TSF app will aid the designer through visualization of components needed at any time-point in the story through a drag-and-drop approach. In brief, the TSF app will support the transreality storyboarding process, to the extent that non-expert designers may choose the story (type of experience response desired) and the app may help them through suggested inputs.

An example of a user experience of someone creating a Transreality story structure on a mobile app is shown in Figure 4. This shows a tentative high-level workflow that allows a Transreality story structure to be compiled in a rapid way.

This would begin by launching the app and creating a new TSF project. First steps would be to add tracks relating to realities that will be encountered in the production of the experience, then drill down and map scenes and the shots within them, linking shots from one track with shots from other tracks that they activate connected story content with (refer to the process outlined in Figure 3). The closing part of the process would list in each shot what sensory and interaction elements would be utilised. Additional features, to be added at later time in the app's development, could cater for administrative functions such as affordances to track project assets, team roles and project management timelines.

\section{Concluding Remarks and Future Directions}

The need for media, advertising and marketing efforts to deliver a consistent message across outputs heralded the emergence of Transmedia approaches to resolving issues of incoherence in narrative expressions of promotions. Transmedia productions ensured stories had more synergy between the components expressed in different media. With the advent of immersive technologies such as Augmented and Virtual Reality as well as more multisensory and interactive experiences, there was a need to evolve Transmedia approaches further to maintain coherence not just across different media but also different immersive realities.

Immersive Reality experiences and devices by their very nature can be considered natively Transmedia, so novel meta-Transmedia workflows, such as those that utilise the Transreality Storyboarding Framework, are needed to ensure stories are conveyed coherently across and through such native, a-priori Transmedia channels.

The Transreality Storyboarding Framework can be used to adapt Transmedia marketing, branding and advertising approaches to take advantage of sensory and interactive affordances that have been made available through immersive reality technologies and experiences. Open source tools, such as the mobile app proposed in this paper, can rapidly onboard creative professionals with these new approaches so they can ensure their marketing, advertising and branding campaigns engage consumers and clients. Professionals are also more likely to secure new and repeat customers that raise and, at minimum, sustain diverse income streams. Although developing income streams is a desirable outcome, an additional effect of adopting the Transreality Storyboarding Framework will be advancing novel, engaging and inspiring storytelling that advances the state of the art that creative professionals must stay aligned and up to date with as a matter of continuing professional development.

\section{References}

[1] J. Rasool, J. Molka-Danielsen, and C. H. Smith, "Designing Interfaces for Creative Learning Environments Using the Transreality Storyboarding Framework", In Proceedings of the 2020 2nd International Conference on Big Data Engineering (BDE 2020), Association for Computing Machinery, New York, NY, USA, 2020, 118-125. DOI:https://doi.org/10.1145/3404512.3404530

[2] J. Steuer, "Defining Virtual Reality: Dimensions Determining Telepresence", Journal of Communication, 4 (2), pp 73 - 93, 1992.

[3] J. J. Gibson, Neuromancer. New York: Ace Books, 1984.

[4] F. Biocca, "The Cyborg's Dilemma: Progressive Embodiment in Virtual Environments", Journal of Computer-Mediated Communication, Vol 3, Issue 2, 1997. Online: https://doi.org/10.1111/j.10836101.1997.tb00070.x

[5] M. Slater, "A note on presence terminology." Presence connect, Vol 3. No. 3: 1-5, 2003.

[6] M. Lombard and T. Ditton, "At the Heart of It All: The Concept of Presence" Journal of Computer-Mediated Communication, Vol 3, Issue 2, pp. 1-57, 2006. Online at https://doi.org/10.1111/j.1083-6101.1997.tb00072.x

[7] N. J. Bowman, "The Demanding Nature of Video Game Play", Global Game Jam, West Virginia University, 
Morgantown, WV, January 29, 2016. https://www.youtube.com/watch?v=KFU55HwLcIY

[8] N. J. Bowman, "Editorial: Video Games as Demanding Technologies", Medial and Communication, Vol 7, Issue 4 , pp. 144-148, 2019. ISSN: 2183-2439, DOI:10.17645/mac.v7i4.2684

[9] R. Busselle, H. Bilandzic, "Measuring Narrative Engagement", Media Psychology, 12:4, pp. $321-347$, Dec $2009 . \quad$ Online http://dx.doi.org/10.1080/1521326093287259

[10] G. Pressgrove, N.D. Bowman, "From immersion to intension? Exploring advances in prosocial storytelling" Int J Nonprofit Volunt Set Mark., John Wiley \& Sons, Ltd., pp. 1-12, $2020 . \quad$ Online https://doi.org/10.1002/nvsm.1689

[11] R. Busselle, H. Bilandzic, "Fictionality and Perceived Realism in Experiencing Stories: A Model of Narrative Comprehension and Engagement", Communication Theory, Vol. 18, No. 2, pp. 255 - 280, 2008.

[12] Z. Ma, "Effects of immersive stories on prosocial attitudes and willingness to help: Testing psychological mechanisms. Media Psychology, pp 1-26, 2019. Online http://doi.org/10.1080/15213269.2019.1651655

[13] H. Jenkins, "Transmedia Storytelling." Technology Review, 2003, Accessed 03.12.19, Online: http://www.technologyreview.com/biomedicine/13052

[14] H. Jenkins, Convergence Culture: Where Old and New Media Collide, New York University Press, New York University, 2006.

[15] C. Dena, "Transmedia Practice: Theorizing the Practice of Expressing a Fictional World Across Distinct Media and Environments" PhD thesis. Sydney: University of Sydney, 2009.

[16] R. Pratten, Getting Started in Transmedia Storytelling 2nd Edition, CreateSpace Independent Publishing Platform; (19 Aug. 2015)

[17] R. O’Meara, A. Bevan, “Transmedia Theory's Author Discourse and Its Limitations" Journal of Media Culture, Vol 21, No 1, 2018. Online: http://journal.mediaculture.org.au/index.php/mcjournal/article/view/1366 Accessed: 03.12.19.

[18] N. Lambert, and J. Rasool, "Leonardo Da Vinci and Perpetual Motion", Victoria and Albert Museum, London, UK. London Design Week, September 2019, https://www.londondesignfestival.com/sites/default/files /ldf19_guide_web-use_2019.09.17-compressed.pdf

[19] A. Bernardoni, "Leonardo Da Vinci and Perpetual Motion”, Museo Galileo, Florence, Italy, 2019. https://www.museogalileo.it/en/museum/explore/tempor ary-exhibitions/1802-leonardo-da-vinci-and-perpetualmotion.html

[20] C. Wang, "Using Transmedia Storytelling and Marketing to Increase Engagement with the David Owsley Museum of Art", Master of Arts Final Project submission, Ball State University, Indiana, USA, 2018.

[21] P. E. Sellars, "Dissent and Disruption: How Artists Redefine Museum Spaces and Audience Engagement". MAD-RUSH, Undergraduate Research Conference, 2019. https://commons.lib.jmu.edu/madrush/2019/dissent/1

[22] C. H. Smith, "Context Engineering Experience Framework", Electronic Visualisation and the Arts, British Computer Society. 191-192, 2016.

[23] Peltz Gallery, (2019). Leonardo da Vinci and Perpetual Motion: Visualising Impossible Machines, Peltz Gallery, London, UK. http://www.bbk.ac.uk/linguistics/arts/research/peltzgallery/peltz-gallery-exhibitions-andevents/Visualising_Impossible_Machines

[24] WEKIT Project, (2017). Accessed 17.12.19 at http://wekit.eu

[25] F. Wild, (Ed.). "Affordances for Capturing and Reenacting Expert Performance with Wearables", Fridolin Wild et al, 12th European Conference on Technology Enhanced Learning, ECTEL 2017, http://wekit.eu/affordances-capturing-re-enactingexpert-performance-wearables/

[26] Hobs 3D Academy, Accessed 04.06.20 at https://hobs3d.com/services/hobs-academy

[27] J. Molka-Danielsen, "Exploring the Role of Virtual Worlds in the Evolution of a Co-creation Design Culture" H. Salmela and A. Seli (Eds.), SCIS 2011, LNBIP 86, Springer-Verlag Berlin Heidelberg, pp. 3-15, 2011.

[28] B.J. Pine II, J. H. Gilmore, "The Experience Economy". Harvard Business School Press, Boston, 1999.

[29] Odyssey Works, Accessed 04.06.20 at http://www.odysseyworks.org/

[30] Waldorf Project, Accessed 05.06.20 at https://waldorfproject.com/

[31] Co-Reality Collective, Accessed 07.06.20 at https://coreality.co/

[32] G. D. Lavold, "Developing and using the work breakdown structure," in Cleland, D. I. and King W. R. (Eds.), Project Management Handbook, 2nd ed. New York: Van Nostrand Reinhold, 1988, pp. 302-323. 